\title{
146. Associations between muscle myotonometry (a)symmetry and functional movement performance in young women and men
}

\author{
Kęstutis Radžiūnas ${ }^{1}$, Margarita Radžiūniené ${ }^{2}$, Alfonsas Vainoras ${ }^{3}$, Jonas Poderys ${ }^{4}$ \\ $1,2,3,4$ Lithuanian University of Health Sciences, Institute of Sports, Kaunas, Lithuania \\ ${ }^{4}$ Lithuanian Sports University, Institute of Sport Science and Innovations, Kaunas, Lithuania \\ ${ }^{1}$ Corresponding author \\ E-mail: ${ }^{1}$ kestutis.radziunas@gmail.com, ${ }^{2}$ margarita.radziuniene@gmail.com, ${ }^{3}$ alfavain@gmail.com, \\ 4jonas.poderys@lsu.lt
}

Received 5 December 2017; received in revised form 24 January 2018; accepted 2 February 2018 DOI https://doi.org/10.21595/jme.2018.19686

Check for updates

\begin{abstract}
Asymmetry of body parts refers to absence of symmetry and unevenly developed body parts in terms of the axis and plane. It has been long ago noticed that no perfectly correct human body exists, and bodies are to a greater or lesser extent asymmetric. A passive way of life, insufficient movement and inappropriate ergonomics of the workplace have a negative impact on the skeletal muscle system, usually resulting in reduced muscle power, static and dynamic muscle endurance, and altered muscle tone. The aim is to determine whether muscle myotonometry parameters are informative in early risk diagnostics to predict the likelihood of skeletal muscle system trauma. The study involved 382 people aged 18-24. Participants were devided into two groups: according to the accumulated composite score. Testing was made one time. The study consisted of functional movement and myotonometry evaluation. The study analyses and presents comparisons of the results depending on the trauma likelihood and sex. In addition, asymmetry of the left and the right body side is investigated. The analysis of the asymmetries (delta) in the frequency of damped oscillations of trapezius, biceps femoris, gastrocnemius (inner and outer part) on the left and right sides of the body demonstrated greater asymmetries between the left and the right sides of the body in the groups of both men and women with a lower chance of trauma. The assessment of asymmetries in femoris rectus and tibialis anterior between the left and the right sides of the body showed differences in the frequency of damped oscillations in all groups. Muscle myotonometry parameters are informative and may be used in early risk diagnostics to predict skeletal muscle system trauma. The asymmetry in tibialis muscle tone between the left and the right leg causes poorer parameters of functional movement performance in women. No associations were found to exist with the ability to perform functional movements in men. The asymmetry in femoris muscle tone between the left and right leg demonstrates the ability of young subjects to perform functional movements. It is characteristic of men and, to a lower extent, of women.
\end{abstract}

Keywords: physical function, functional performance, fitness, motor control.

\section{Introduction}

Body symmetry is primarily understood as a proportionate distribution of equal body parts or forms. From the biomechanical and clinical perspective, symmetric body posture is a physical position of a person when seated, standing or moving, which allows body segments to remain in one biomechanical chain, and due to coordinated activity of the segment, to overcome gravitation and acceleration forces $[1,2]$. Normal functioning of the skeletal muscle system allows our body to maintain balance without additional activity of muscles; however, occurring asymmetric changes in the skeletal muscle system result in fatigue and deformation of body structural parts $[3,4]$.

Asymmetry of body parts refers to absence of symmetry and unevenly developed body parts in terms of the axis and plane [5]. It has been long ago noticed that no perfectly correct human body exists and bodies are to a greater or lesser extent asymmetric. A passive way of life, 
insufficient movement and inappropriate ergonomics of the workplace have a negative impact on the skeletal muscle system, usually resulting in reduced muscle power, static and dynamic muscle endurance, and altered muscle tone [6-8]. All asymmetries of the neuromuscular systems result in the occurrence of scoliosis, changes of spinal kyphosis and lordosis as well as physiological disorders and functional injuries of the thorax [9].

The muscle has active and passive mechanical features.

Muscle tone is a mechanical tension of the muscle regardless of its conscious contraction $[10,11]$. Muscle tone has been noticed to reflect muscle recovery characteristics after mechanical effects [11].

Muscle elasticity (resilience, viscosity) is the ability of the muscle to return to the previous state upon disappearance of external forces affecting the muscle [12]. Muscle elasticity reflects the state of blood circulation when the muscle works. When a person moves and muscles contract, blood is constantly supplied to the cells of the muscle [13]. Contraction of the muscle is constantly accompanied by muscle relaxation during which blood can flow easily; therefore, the muscle has to recover to its natural state over a short period, and muscle elasticity reflects this $[14,15]$. Muscle elasticity is characterised by logarithmic decrement of oscillations, i.e., the coefficient of elasticity. It has been noticed that the greater the logarithmic decrement of damped oscillations, the lower the muscle elasticity [16-18].

Muscle stiffness refers to the ability of the muscle to resist the deforming forces $[14,15]$. Increased muscle stiffness means that its antagonists receive additional load in order to maintain appropriate strength distribution, which determines an increase in energy input to perform movements $[19,20]$. And vice versa, a decrease in muscle stiffness results in reduced antagonist resistance [21]. Muscle stiffness refers to a state of the muscle conditioned by its resistance to compression and stretching [22].

The interaction of muscle tone, elasticity and stiffness ensures joint stability when movement is performed [23]. These components are also considered to provide the most substantial protection in maintaining the joint stable in the presence of unexpected interferences [24, 25].

Early diagnosis of structural changes in the skeletal muscle system in young subjects may help to avoid trauma in everyday activities and sports as well as functional and physical disorders in future.

\section{Materials and methods}

For the research, permission of Kaunas Regional Biomedical Research Ethics Committee (No. BE-2-46, as of 07/10/2014) was obtained. The study involved all first-year students (aged 18-24) enrolled in the integral study programme Medicine at the Lithuanian University of Health Sciences during 2014-2015. Of the respondents who agreed to participate in the study $(n=423), 382$ were selected, and 35 did not match the selection criteria. The participants were selected according to the following selection criteria: age - 18-24 years; right-handed; BMI - 16-29.9; health certificate approving the subject's ability to participate in physical activity; voluntary agreement to participate in the study; and filling in the questionnaire. The exclusion criteria were as follows: a trauma of the skeletal muscle system during the past 12 months, and active athletes professionally aiming at sports results and participating in sports competitions.

The study analyses and presents comparisons of the results depending on the trauma likelihood and sex. In addition, asymmetry of the left and the right body side is investigated.

All subjects performed 7 functional movement assessment tasks and were divided into 2 groups according to the accumulated composite score. Later, all subjects participated in the study assessing their muscle tone parameters.

Functional movement assessment. Movement quality was investigated based on Cook's criteria assessed on the scale from 0 to 3 points [26]. Of 7 assessed functional movements, 5 are symmetric, i.e., they are performed with both the left and the right side of the body; therefore, the movements were assessed separately for both sides. The subjects performed the following 
movements: deep squat, hurdle step, in-line lunge, shoulder mobility, active straight leg raise, trunk stability push-up and rotary stability. Following many authors [27-29], the reference point is as follows: subjects with the score $\leq 14$ have a greater chance of trauma (Group 1), and those with the score $\geq 15$ have a lower chance of trauma (Group 2). In case of different evaluation scores for the left and the right body sides, the subjects were attributed to the groups according to the lower score [30].

Muscle myotonometry parameters were recorded using myotonometer Myoton-3, which measures frequency and amplitude values of damped oscillations, where damped oscillation frequency characterizes tone, and logarithmic decrement of oscillation characterizes elasticity of tissues. The software of the device allows obtaining generalized values of many measurements and gives the stiffness coefficient of damped oscillations on the basis of measured relative values of frequency and amplitude of damped oscillations. This stiffness coefficient gives evidence of the resistance of tissues to the deforming forces [31, 32].

The study analysed the parameters of the muscle tone of the trapezius, biceps femoris, gastrocnemius (inner and outer part), rectus femoris and tibialis anterior on the right and the left side of the body. The subjects had to lie down relaxed on a couch on the abdomen with their arms placed beside their body and palms up. First, the parameters of trapezius were measured on the left and the right side of the body; then, biceps femoris and the outer part as well as the inner part of gastrocnemius on the left side of the body were measured. After that, the muscles on the right side of the body were measured in the same order. When the subjects lay relaxed on their back, rectus femoris and tibialis anterior of the left leg were measured, followed by measurement of rectus femoris and tibialis anterior of the right leg.

Statistics and data analysis. The statistical data analysis was performed using the IBM SPSS (Statistical Package for Social Sciences) 22.0 for Windows and Microsoft Office Excel 2010 statistical analysis packages.

The differences were considered statistically significant when $p<0.05$. The bars in figures provide medians and confidence intervals $(\mathrm{CI})$.

\section{Results}

There were no statistically significant differences between the subjects in groups in terms of sex, age, height, body weight and physical activity (Table 1).

Table 1. Distribution of the subjects according to the functional movement assessment results (data are presented as $\left.x_{m e}(C I)\right)$

\begin{tabular}{|c|c|c|c|c|}
\hline \multicolumn{2}{|c|}{ Group } & I group $(n=253)$ & II group $(n=129)$ & Significant level \\
\hline \multirow{2}{*}{ Sex $(\mathrm{n})$} & Men & $81(32 \%)$ & $37(29 \%)$ & \multirow{2}{*}{ Chi $^{2}=0.445 ; p=0.292$} \\
\cline { 2 - 5 } & Women & $172(68 \%)$ & $92(71 \%)$ & \\
\hline \multirow{2}{*}{ Age $(\mathrm{yr})$} & Men & $19.00(18.83-19.69)$ & $19.00(18.96-19.29)$ & $U=968.0 ; p=0.747$ \\
\cline { 2 - 5 } & Women & $19.00(18.95-19.18)$ & $19.00(18.90-19.14)$ & $U=2951.5 ; p=0.837$ \\
\hline \multirow{2}{*}{ Height $(\mathrm{m})$} & Men & $1.83(1.82-1.86)$ & $1.81(1.80-1.83)$ & $U=813.0 ; p=0.128$ \\
\cline { 2 - 5 } & Women & $1.68(1.67-1.69)$ & $1.66(1.65-1.69)$ & $U=793.5 ; p=0.094$ \\
\hline \multirow{2}{*}{ Weight $(\mathrm{kg})$} & Men & $76.00(74.01-81.34)$ & $71.50(69.70-75.96)$ & $U=813.0 ; p=0.128$ \\
\cline { 2 - 5 } & Women & $62.00(61.56-65.25)$ & $58.00(55.80-61.42)$ & $U=2922.0 ; p=0.477$ \\
\hline \multirow{2}{*}{ BMI $\left(\mathrm{kg} / \mathrm{m}^{2}\right)$} & Men & $22.99(22.09-23.86)$ & $21.74(21.29-22.92)$ & $U=836.0 ; p=0.183$ \\
\cline { 2 - 5 } & Women & $21.77(21.76-22.92)$ & $21.11(20.02-21.71)$ & $U=2860.5 ; p=0.561$ \\
\hline
\end{tabular}

The subjects who scored $\leq 14$ in performing functional movement tests were attributed to Group 1 and were considered to have a greater chance of trauma (the composite score for women was 13.00 (12.41-12.82) and that for men was 13.00 (11.99-12.81)). The subjects who scored $\geq 15$ were attributed to Group 2 and were considered to have a lower chance of trauma (the composite score for women was 15.00 (15.47-16.17) and that for men was 16.00 (15.56-16.14)) [27-29, 33].

Parameters of muscle myotonometry. The three parameters assessed were the frequency of 
damped oscillations (muscle tone), the logarithmic decrement of damped oscillations (muscle elasticity), and the stiffness coefficient of damped oscillations (muscle agonist and antagonist imbalance ratio).

\subsection{Frequency of damped oscillations}

The women in the group with a greater chance of trauma had a statistically significant difference between gastrocnemius (inner part), rectus femoris and tibialis anterior on the left and the right side of the body. In the women with a greater chance of trauma, the median of the frequency of damped oscillations in rectus femoris of the left leg was $11.0 \mathrm{~Hz}$ and that of the right leg was $11.5 \mathrm{~Hz}$. In the women with a lower chance of trauma, a statistically significant difference in the frequency of damped oscillations was observed between rectus femoris of the left leg $(11.1 \mathrm{~Hz})$ and the right leg $(11.6 \mathrm{~Hz})$. There were no differences in the frequency of damped oscillations of other analysed muscles between the women in Group 1 and Group 2 (Fig. 1).

In the men with a greater chance of trauma, a statistically significant difference was determined in biceps femoris between the left and the right side of the body: the median of the frequency of damped oscillations on the left leg was $14.6 \mathrm{~Hz}$ and that on the right leg was 14.7. Meanwhile, in the men with a lower chance of trauma, the median of the frequency of damped oscillations in femoris rectus was $11.8 \mathrm{~Hz}$ on the left leg and $12.7 \mathrm{~Hz}$ on the right leg. Regardless of the chance of trauma, the frequency of damped oscillations of other analysed muscles in the men was similar (Fig. 2).

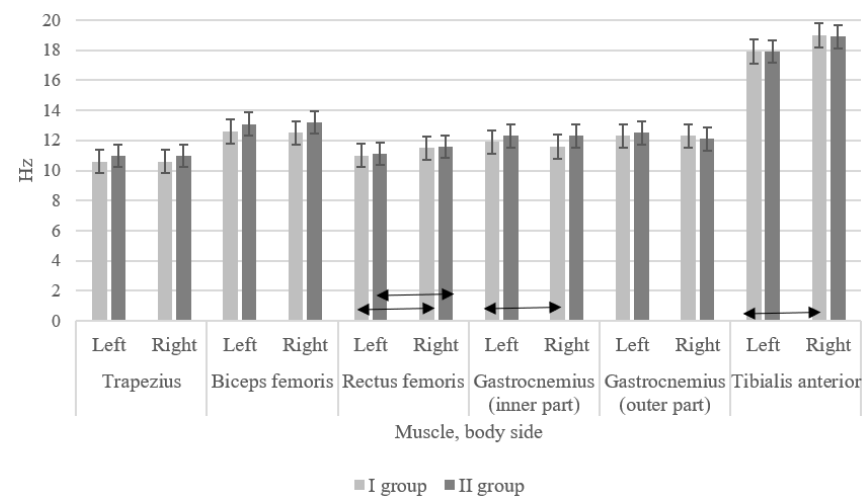

Fig. 1. Data of muscle tone (frequency of damped oscillations, Hz) in groups of women; $\leftrightarrow-$ statistically significant difference comparing the results between both sides of the body and between the groups

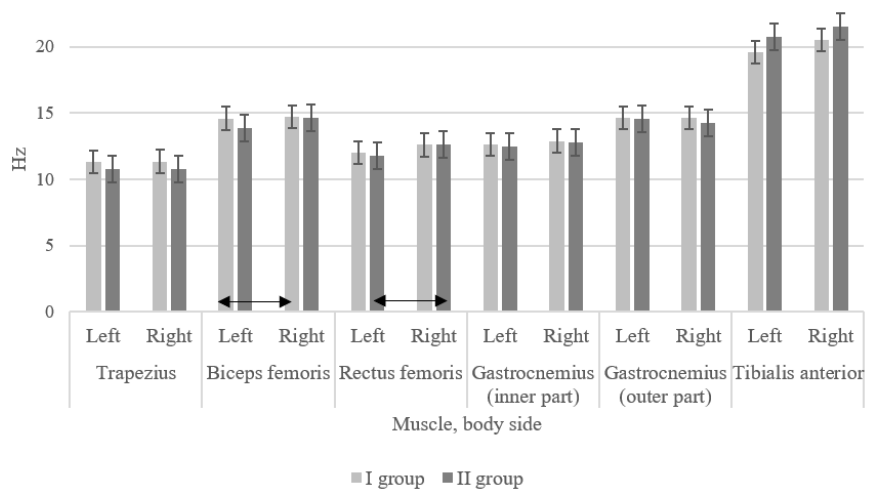

Fig. 2. Data of muscle tone (frequency of damped oscillations, Hz) in groups of men; $\leftrightarrow-$ statistically significant difference comparing the results between both sides of the body and between the groups 


\subsection{Logarithmic decrement of damped oscillations of the muscle}

The analysis of the results of the logarithmic decrement of damped oscillations in the women with a greater chance of trauma demonstrated a statistically significant difference between the right and the left side of the body in terms of trapezius, biceps femoris, gastrocnemius (inner part) and tibialis anterior. In the women with a greater chance of trauma, the logarithmic decrement of damped oscillations was similar in all the analysed muscles on both the left and the right side of the body. In the women with a greater chance of trauma, a lower logarithmic decrement of damped oscillations was determined in trapezius of the right side of the body in comparison with the women with a lower chance of trauma. The logarithmic decrement of damped oscillations of other analysed muscles was similar in the groups of the women regardless of a greater or lower chance of trauma (Fig. 3).

In the men with a greater chance of trauma, the logarithmic decrement of damped oscillations of gastrocnemius (inner part) was greater on the left side of the body. However, in the men with a lower chance of trauma, the logarithmic decrement of damped oscillations of gastrocnemius (outer part) was greater on the right side of the body. The logarithmic decrement of damped oscillations of gastrocnemius (outer part) on the left side of the body was lower in the men with a lower chance of trauma in comparison with the men with a greater chance of trauma. There were no statistically significant differences in the logarithmic decrement of damped oscillations of other analysed muscles (Fig. 4).

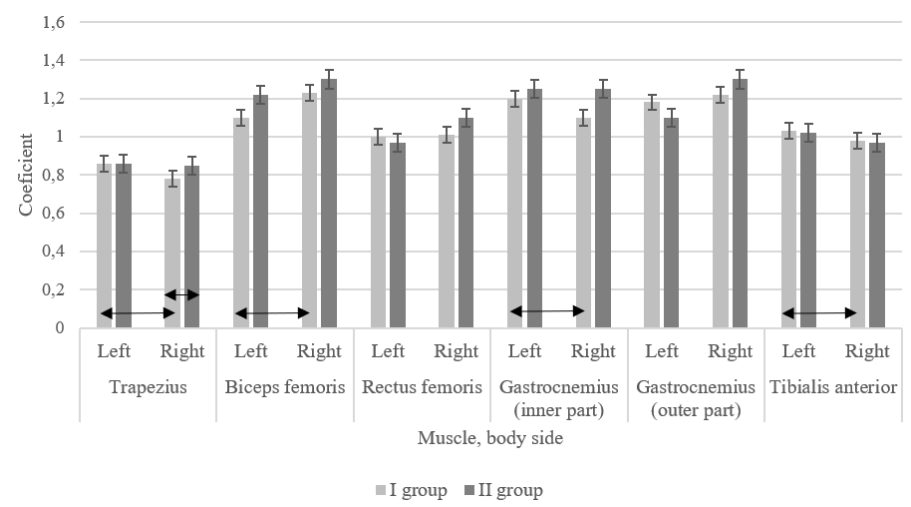

Fig. 3. Data of muscle elasticity (logarithmic decrement of damped oscillations) in groups of women; $\leftrightarrow-$ statistically significant difference comparing the results between both sides of the body and between the groups

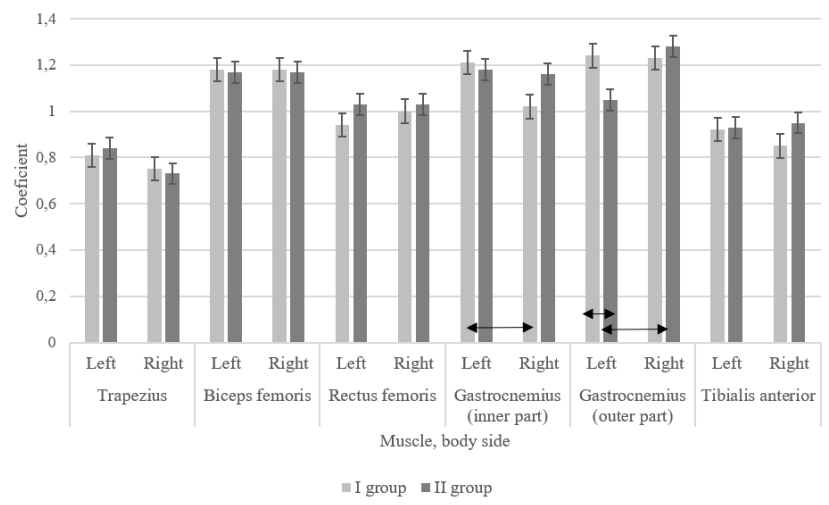

Fig. 4. Data of muscle elasticity (logarithmic decrement of damped oscillations) in groups of men; $\leftrightarrow-$ statistically significant difference comparing the results between both sides of the body and between the groups 


\subsection{Stiffness coefficient of damped oscillations of the muscle}

In the women with a greater chance of trauma, the stiffness coefficients of damped oscillations in trapezius, gastrocnemius (outer part) and tibialis anterior were statistically significantly different between the left and the right side of the body. In the women with a lower chance of trauma, the stiffness coefficients of damped oscillations of all the assessed muscles were similar regardless of the side of the body. The analysis of the data on the stiffness coefficients of damped oscillations in all the muscles selected for the research demonstrated that there were no statistically significant differences between the groups of the women (Fig. 5). It was determined that the stiffness coefficients of damped oscillations of muscles in the groups of the men were not statistically significantly different in terms of the body side and the chance of trauma.

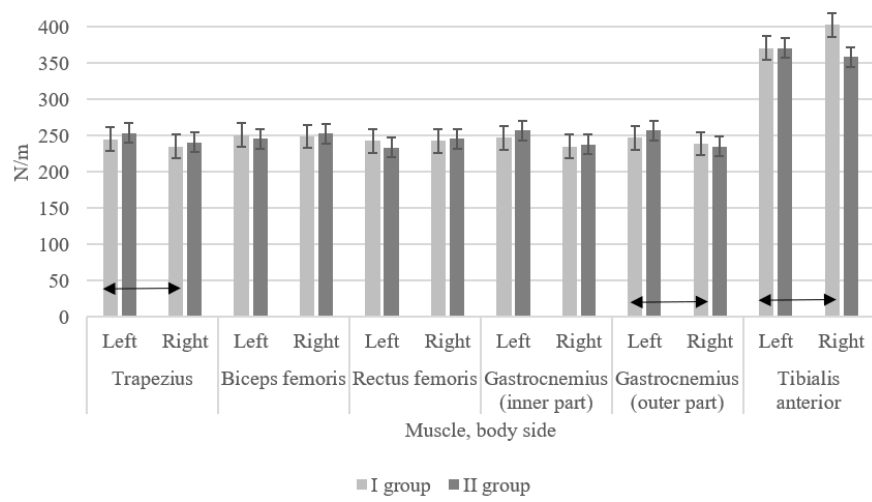

Fig. 5. Data of muscle stiffness (stiffness coefficient of damped oscillations, $N / m$ ) in groups of women; $\leftrightarrow-$ statistically significant difference comparing the results between both sides of the body and between the groups

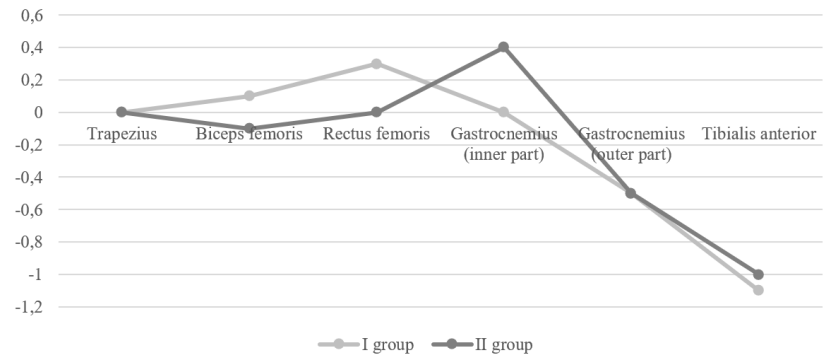

a) Women

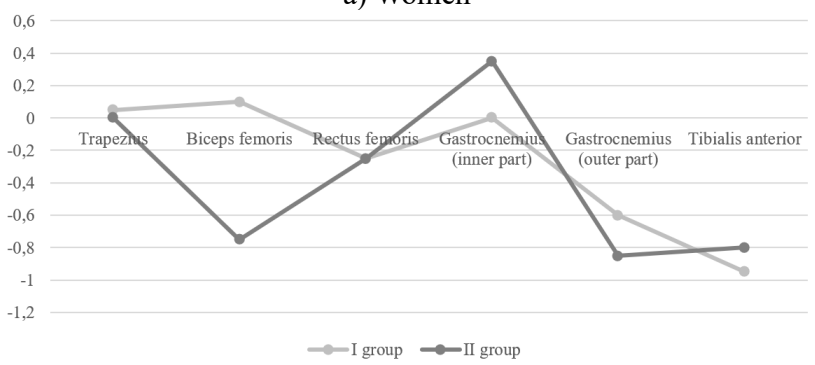

b) Men

Fig. 6. Peculiarities of body muscle asymmetry in young subjects who are less or more likely to experience trauma: a) women, b) men

The analysis of the asymmetries (delta) in the frequency of damped oscillations of trapezius, biceps femoris, gastrocnemius (inner and outer part) on the left and right sides of the body 
demonstrated greater asymmetries between the left and the right sides of the body in the groups of both men and women with a lower chance of trauma. The assessment of asymmetries in femoris rectus and tibialis anterior between the left and the right sides of the body showed differences in the frequency of damped oscillations in all the 4 groups (Fig. 6).

\section{Discussion}

Muscles are characterized by active and passive mechanical features. Active mechanical features of the muscle refer to the dependence of strength developed by innervated muscle fibers on the length of the muscle, speed and time of contraction of the muscle [12, 34]. Passive mechanical features of the muscle are characterized by muscle tone, elasticity, and stiffness $[14,15]$. In the normal state, the muscle is resilient. Its resilience is conditioned by involuntary tension and readiness to resist the external forces, i.e., stretching $[11,35]$.

The tone of the muscle refers to a mechanical tension independent of the voluntary contraction of the muscle $[10,11]$. The muscle tone has been considered to reflect the capability of the muscle to recover after mechanical effects [11]. An increased muscle tone demonstrates an increase in the internal tension, which leads to poorer blood circulation and metabolism conditioning pain, injuries and overload [37]. A reduced muscle tone results in reduced muscle efficiency and weakening of the muscle function $[7,8]$. The muscle tone is most probably best described by the frequency of damped oscillations $(\mathrm{Hz})$, when oscillations fade following a sudden mechanical effect. The greater the muscle tone, the more tense the muscle resulting in the greater frequency of damped oscillations [38, 39].

The results of our study demonstrated that the parameters of muscle myotonometry were informative and might be used in early diagnostics of skeletal muscle system trauma. In this research, we analysed three features of muscle elasticity in subjects with a greater or lower chance of trauma: frequency of damped oscillations of the muscle defining the muscle tone; logarithmic decrement of damped oscillations defining the muscle elasticity; and muscle stiffness defining muscle stasis.

The analysis of the tone and elasticity features of gastrocnemius (inner part) showed that the frequency of damped oscillations and the logarithmic decrement in the women with a greater chance of trauma were statistically significantly different in comparison with the women with a lower chance of trauma. In addition, our study determined a statistically significant difference in the logarithmic decrement of damped oscillations of the inner part of gastrocnemius between the left and the right leg in the men of Group 1. Therefore, we may claim that poorer parameters of functional movement performance in young subjects are conditioned by asymmetry of the calf muscle tone and elasticity between the right and left leg.

In a similar study conducted by Vain et al., elastic muscle characteristics were measured in men in the lying position (as in our study). The researchers found that the frequency of damped oscillations was $13.0 \mathrm{~Hz}$, and the logarithmic decrement of damped oscillations was 1.28. The authors did not differentiate between the right and left leg parameters [40]. According to the results of the two studies, both the frequency of damped oscillations and the logarithmic decrement of damped oscillations were similar. The analysis of stiffness coefficients of damped oscillations in our study did not show statistically significant differences between the groups. The researchers who tested the inner part of gastrocnemius in subjects in the lying position (with muscles relaxed) observed that the stiffness coefficient of damped oscillations of the muscle was $204.1 \mathrm{~N} / \mathrm{m}$ [40]. The data confirm that stiffness coefficients of damped oscillations in muscles of our subjects were greater. This might be explained by the fact that the authors of the above-mentioned study focused more not only on testing the muscles in the lying position, but also on the comparison of the results when the subjects were lying and standing on stable and unstable surfaces. We did not test the muscles in other positions, although this may be our future research, because the results of studies conducted by other researchers demonstrated that both the frequency of damped oscillations and the muscle stiffness coefficient of damped oscillations changed, i.e., were greater when muscles 
were tested in the standing position on both the stable and the unstable surface. The logarithmic decrement of damped oscillations remained similar. The mentioned authors studied the differences between muscle elasticity when the subjects were standing on the stable and the unstable surface. They found that there was no statistically significant difference between the frequency of damped oscillations, the stiffness coefficient of damped oscillations and the logarithmic decrement of damped oscillations when the subjects were standing on different surfaces [40].

According to Cupriks et al., the frequency of damped oscillations of muscles in young men weightlifters should be 11-16 Hz, the logarithmic decrement of damped oscillations should be 1.0-1.2, and the stiffness coefficient of damped oscillations should be $150-300 \mathrm{~N} / \mathrm{m}$ regardless of the muscle tested [41]. In our study, we assessed the minimal and the maximal values of all the analysed muscles and found that the frequency of damped oscillations varied between 9.3 and $23.0 \mathrm{~Hz}$, the logarithmic decrement of damped oscillations varied between 0.6 and 1.6, and the stiffness coefficient of damped oscillations was 288-445.5 N/m. The differences found between our research results and those of Latvian researchers may be due different subjects; besides, in the Latvian study, the researchers did not indicate which particular muscles were assessed.

Mullix et al., in a study conducted in Great Britain, tested healthy men (mean age, 25.7 years). In this study, the researchers assessed the elastic features of rectus and biceps femoris. The subjects had to abstain from alcohol for 48 hours. Testing was performed when the subjects had been lying on the back for $10 \mathrm{~min}$. Testing of rectus femoris showed that the frequency of damped oscillations was $15.50 \mathrm{~Hz}$. The researchers did not differentiate between the results of the right and the left leg [42]. In our study, we analysed the elastic features of the left and the right leg in both groups of women and men. The obtained results demonstrated that the frequency of damped oscillations of the right leg muscles in both groups of women was greater; meanwhile, among men, the frequency of damped oscillations of rectus femoris was greater only for the right leg in Group 2. The comparison of the results of women and men in 2 groups did not yield statistically significant differences. Hence, the results of the authors obtained in the study in Great Britain are similar to ours.

In analysing the logarithmic decrement of damped oscillations of rectus femoris in young men, the authors found that it was 1.32; the stiffness coefficient of damped oscillations was $279 \mathrm{~N} / \mathrm{m}$ [42]. The comparison of our results and those of other researchers shows that the logarithmic decrement of damped oscillations of rectus femoris was different, but this may be explained by the fact that the mentioned authors analysed the logarithmic decrement of damped oscillations together for the left and the right leg.

In the study conducted in Great Britain, the researchers analysed elastic features of relaxed biceps femoris and found that the frequency of damped oscillations was $16.2 \mathrm{~Hz}$, the logarithmic decrement of damped oscillations was 1.21, and stiffness coefficient of damped oscillations was $290 \mathrm{~N} / \mathrm{m}$ [42]. The frequencies of damped oscillations of rectus femoris in this study and in our study differ. However, the comparison of their and our results in terms of the logarithmic decrement of damped oscillations shows similarity. The analysis of the stiffness coefficient of damped oscillations of the muscles in our study subjects demonstrates that the stiffness coefficient of damped oscillations in the study in Great Britain was higher, which may be due to the fact that the researchers tested 20 healthy men with the mean age 25.7 years, i.e., older than the subjects in our study [42].

It is well known that the capacity of muscles is the condition of their full function, which reduces the likelihood of trauma. Mersmann et al. studied the stiffness coefficient of damped oscillations in professional volleyball players teenagers over the period of 2 years. The obtained results demonstrated that the strength of leg muscles and stiffness of tissues surrounding the patella changed, which may be the cause of a fine muscle function and a low chance of trauma [43]. The authors claim that an increase in the stiffness coefficient of damped oscillations means lower chances of trauma. In our study, we determined that the stiffness coefficients of damped oscillations of analysed muscles were similar in groups with a greater and a lower chance of trauma. 
Cupriks et al. studied changes in elastic features of muscles in young men (20 years old) who performed various functional movements. The researchers tested muscles three times: before performance of functional movements, after functional movements, and after stretching exercises of the muscle with the highest workload. The results obtained demonstrated that the parameters of the muscle tone (frequency of damped oscillations and stiffness of damped oscillations) changed during functional movements and after stretching exercises; however, the elastic features of the muscle were different only when specific functional movements were performed. Unfortunately, the authors did not indicate the specific movements analysed [41]. The authors came to a conclusion that a greater stiffness of damped oscillations and a greater frequency of damped oscillations determine a smaller amplitude of movements. The results obtained in our study confirm this conclusion, as statistically significant correlations were established between the frequency of damped oscillations, stiffness of damped oscillations and functional movements of some muscles. The greater the frequency of damped oscillations and the greater the stiffness coefficient of damped oscillations, the lower the results of functional movement assessment.

We found a statistically significant difference in the frequency of damped oscillations of rectus femoris between the left and the right leg in the women with a greater and a lower chance of trauma and in the men with a lower chance of trauma. Similar results were obtained by other authors who also noted differences in the frequency of damped oscillations, the logarithmic decrement of damped oscillations and the stiffness coefficient of damped oscillations of rectus femoris between the left and the right leg [17]. Thus, asymmetry of the femoris muscle tone between the left and the right leg is related to the ability of the subject to perform functional movements.

\section{Conclusions}

1) Muscle myotonometry parameters are informative and may be used in early risk diagnostics to predict skeletal muscle system trauma.

2) The asymmetry in tibialis muscle tone between the left and the right leg causes poorer parameters of functional movement performance in women. No associations were found to exist with the ability to perform functional movements in men.

3) The asymmetry in femoris muscle tone between the left and right leg demonstrates the ability of young subjects to perform functional movements. It is characteristic of men and, to a lower extent, of women.

\section{References}

[1] Aruin A., Kanekar N. Effect of a textured insole on balance and gait symmetry. Experimental Brain Research, Vol. 231, Issue 2, 2013, p. 201-208.

[2] Gambelli C., Theisen D., Willems P., Schepens B. Motor control of landing from a jump in simulated hypergravity. PLoS One, 2015, https://doi.org/10.1371/journal.pone.0141574.

[3] Blaszczyk J., Beck M., Sadowska D. Assessment of postural stability in young healthy subjects based on directional features of posturographic data: vision and gender effects. Acta Neurobiologiae Experimentalis (Wars), Vol. 74, Issue 4, 2014, p. 433-442.

[4] Kazennikov O., Kireeva T., Shlykov V. Influence of the movable support under one leg on human vertical posture during standing with asymmetric load on legs. Fiziologiia Cheloveka, Vol. 40, Issue 3, 2014, p. 57-65.

[5] Yagi M., Ohne H., Konomi T., Fujiyoshi K., Kaneko S., Takemitsu M., et al. Walking balance and compensatory gait mechanisms in surgically treated patients with adult spinal deformity. The Spine Journal, Vol. 17, Issue 3, 2017, p. 409-417.

[6] Dejanovic A., Harvey E., Mcgill S. Changes in torso muscle endurance profiles in children aged 7 to 14 years: reference values. Archives Physical Medicine and Rehabilitation, Vol. 93, Issue 12, 2012, p. $2295-2301$. 
[7] Caiafa R., Orsini M., Felicio L., Puccioni Sohler M. Muscular weakness represents the main limiting factor of walk, functional independence and quality of life of myelopathy patients associated to HTLV-1. Arquivos de Neuro-Psiquiatria, Vol. 74, Issue 4, 2016, p. 280-286.

[8] Ruscello B., Tozzo N., Briotti G., Padua E., Ponzetti F., D'ottavio S. Influence of the number of trials and the exercise to rest ratio in repeated sprint ability, with changes of direction and orientation. The Journal of Strength and Conditioning Research, Vol. 27, Issue 7, 2013, p. 1904-1919.

[9] Ramsay J., Seoud L., Barchi S., Cheriet F., Joncas J., Turgeon I., et al. Assessment of breast asymmetry in adolescent idiopathic scoliosis using an automated 3D body surface measurement technique. Spine Deformity, Vol. 5, Issue 3, 2017, p. 152-158.

[10] Agyapong Badu S., Warner M., Samuel D., Stokes M. Measurement of ageing effects on muscle tone and mechanical properties of rectus femoris and biceps brachii in healthy males and females using a novel hand-held myometric device. Archives of Gerontology and Geriatrics, Vol. 62, 2016, p. 59-67.

[11] Loturco I., Gil S., Laurino C., Roschel H., Kobal R., Cal Abad C., et al. Differences in muscle mechanical properties between elite power and endurance athletes: a comparative study. The Journal of Strength and Conditioning Research, Vol. 29, Issue 6, 2015, p. 1723-1728.

[12] Wheeler J. B., Mukherjee R., Stroud R. E., Jones J. A., Ikonomidis J. S. Relation of murine thoracic aortic structural and cellular changes with aging to passive and active mechanical properties. Journal of the American Heart Association, Vol. 4, Issue 3, 2015, p. e001744.

[13] Kennedy Lydon T., Crawford C., Wildman S., Peppiatt Wildman C. Renal pericytes: regulators of medullary blood flow. Acta Physiologica, Vol. 207, Issue 2, 2013, p. 212-225.

[14] Eby S., Cloud B., Brandenburg J., Giambini H., Song P., Chen S., et al. Shear wave elastography of passive skeletal muscle stiffness: influences of sex and age throughout adulthood. Clinical Biomechanics, Vol. 30, Issue 1, 2015, p. 22-27.

[15] Huang S., Hsieh C., Lin J., Chen H. Optimal scoring methods of hand-strength tests in patients with stroke. International Journal of Rehabilitation Research, Vol. 34, Issue 2, 2011, p. 178-180.

[16] Mustalampi S., Ylinen J., Kautiainen H., Weir A., Häkkinen A. Acute effects of cold pack on mechanical properties of the quadriceps muscle in healthy subjects. Physical Therapy in Sport, Vol. 13, Issue 4, 2012, p. 265-269.

[17] Aird L., Samuel D., Stokes M. Quadriceps muscle tone, elasticity and stiffness in older males: reliability and symmetry using the MyotonPRO. Archives of Gerontology and Geriatrics, Vol. 55, Issue 2, 2012, p. e31-9.

[18] Fabre J., Martin V., Borelli G., Theurel J., Grélot L. Effects of string stiffness on muscle fatigue after a simulated tennis match. Journal of Applied Biomechanics, Vol. 30, Issue 3, 2014, p. 401-406.

[19] Wu R., Delahunt E., Ditroilo M., Lowery M., De Vito G. Effects of age and sex on neuromuscularmechanical determinants of muscle strength. Age (Dordr), Vol. 38, Issue 3, 2016, p. 57.

[20] Amiri Khorasani M., Kellis E. Acute effects of different agonist and antagonist stretching arrangements on static and dynamic range of motion. Asian Journal of Sports Medicine, Vol. 6, Issue 4, 2015, p. e26844.

[21] Hirai H., Miyazaki F., Naritomi H., Koba K., Oku T., Uno K., et al. On the origin of muscle synergies: invariant balance in the co-activation of agonist and antagonist muscle pairs. Frontiers in Bioengineering and Biotechnology, Vol. 3, 2015, p. 192.

[22] Masi A., Nair K., Evans T., Ghandour Y. Clinical, biomechanical, and physiological translational interpretations of human resting myofascial tone or tension. International Journal of Therapeutic Massage and Bodywork, Vol. 3, Issue 4, 2010, p. 16-28.

[23] Izzo R., Guarnieri G., Guglielmi G., Muto M. Biomechanics of the spine. Part I: spinal stability. European Journal of Radiology, Vol. 82, Issue 1, 2013, p. 118-126.

[24] Maharaj J., Cresswell A., Lichtwark G. The mechanical function of the tibialis posterior muscle and its tendon during locomotion. Journal of Biomechanics, Vol. 49, Issue 14, 2016, p. 3238-3243.

[25] Bryanton M., Bilodeau M. Postural stability with exhaustive repetitive sit-to-stand exercise in young adults. Human Movement Science, Vol. 49, 2016, p. 47-53.

[26] Cook G., Burton L., Hoogenboom B. Pre-participation screening: the use of fundamental movements as an assessment of function - part 1. North American Journal of Sports Physical Therapy, Vol. 1, Issue 2, 2006, p. 62-72.

[27] Fuller J., Chalmers S., Debenedictis T., Townsley S., Lynagh M., Gleeson C., et al. High prevalence of dysfunctional, asymmetrical, and painful movement in elite junior Australian football players assessed using the functional movement screen. Journal of Science and Medicine in Sport, Vol. 20, Issue 2, 2017, p. 134-138. 
[28] Mokha M., Sprague P., Gatens D. Predicting musculoskeletal injury in national collegiate athletic association division II athletes from asymmetries and individual-test versus composite functional movement screen scores. Journal of Athletic Training, Vol. 51, Issue 4, 2016, p. 276-282.

[29] Beach T., Frost D., Callaghan J. FMS ${ }^{\mathrm{TM}}$ scores and low-back loading during lifting-whole-body movement screening as an ergonomic tool? Applied Ergonomics, Vol. 45, Issue 3, 2014, p. 482-489.

[30] Schneiders A., Davidsson A., Hörman E., Sullivan S. Functional movement screen normative values in a young, active population. International Journal of Sports Physical Therapy, Vol. 6, Issue 2, 2011, p. $75-82$.

[31] Lin K., Huang P., Chen Y., Wu C., Huang W. Combining afferent stimulation and mirror therapy for rehabilitating motor function, motor control, ambulation, and daily functions after stroke. Neurorehabilitation and Neural Repair, Vol. 28, Issue 2, 2014, p. 153-162.

[32] Roja Z., Kalkis V., Vain A., Kalkis H., Eglite M. Assessment of skeletal muscle fatigue of road maintenance workers based on heart rate monitoring and myotonometry. Journal of Occupational Medicine and Toxicology, 2006, https://doi.org/10.1186/1745-6673-1-20.

[33] Frost D., Beach T., Campbell T., Callaghan J., Mcgill S. An appraisal of the functional movement screen $^{\mathrm{TM}}$ grading criteria - is the composite score sensitive to risky movement behavior? Physical Therapy in Sport, Vol. 16, Issue 4, 2015, p. 324-330.

[34] Sierra M., Miana Mena F., Calvo B., Muñoz M., Rodríguez J., Grasa J. On using model populations to determine mechanical properties of skeletal muscle. Application to concentric contraction simulation. Annals of Biomedical Engineering, Vol. 43, Issue 10, 2015, p. 2444-2455.

[35] Schleip R., Naylor I., Ursu D., Melzer W., Zorn A., Wilke H., et al. Passive muscle stiffness may be influenced by active contractility of intramuscular connective tissue. Medical Hypotheses, Vol. 66, Issue 1, 2006, p. 66-71.

[36] Schleip R., Klingler W., Lehmann Horn F. Active fascial contractility: Fascia may be able to contract in a smooth muscle-like manner and thereby influence musculoskeletal dynamics. Medical Hypotheses, Vol. 65, Issue 2, 2005, p. 273-277.

[37] Nielson K., Wulff L., Arentsen T. Muscle tension induced after learning enhances long-term narrative and visual memory in healthy older adults. Neurobiology of Learning and Memory, Vol. 109, 2014, p. $144-150$.

[38] Dietsch A., Clark H., Steiner J., Solomon N. Effects of age, sex, and body position on orofacial muscle tone in healthy adults. Journal of Speech, Language and Hearing Research, Vol. 58, Issue 4, 2015, p. 1145-1150.

[39] Mustalampi S., Häkkinen A., Kautiainen H., Weir A., Ylinen J. Responsiveness of muscle tone characteristics to progressive force production. The Journal of Strength and Conditioning Research, Vol. 27, Issue 1, 2013, p. 159-165.

[40] Vain A., Kums T., Ereline J., Pääsuke M., Gapeyeva H. Gastrocnemius muscle tone, elasticity, and stiffness in association with postural control characteristics in young men. Biomechanics, Vol. 64, Issue 4, 2015, p. 525-534.

[41] Cupriks L., Ciematnieks U., Lescinkis M., Cuprika A. Muscle biomechanical qualities monitoring in heavy athletics. Latvian Academy of Sport Education, 2012, p. 22-27.

[42] Mullix J., Warner M., Stokes M. Testing muscle tone and mechanical properties of rectus femoris and biceps femoris using a novel hand held MyotonPRO device: relative ratios and reliability. Working Papers in Health Sciences, Vol. 1, Issue 1, 2012, p. 1-8.

[43] Mersmann F., Bohm S., Schroll A., Boeth H., Duda G., Arampatzis A. Muscle and tendon adaptation in adolescent athletes: a longitudinal study. Scandinavian Journal of Medicine and Science in Sports, Vol. 27, Issue 1, 2017, p. 75-82. 